\title{
CARACTERIZAÇÃO DO NÚMERO CROMOSSÔMICO EM ESPÉCIES DE ODONTOSTILBE E SERRAPINNUS (CHARACIDAE: CHEIRODONTINAE) DA BACIA DO RIO PARAGUAI, BRASIL
}

\author{
CHROMOSOMIC NUMBER CHARACTERIZATION OF ODONTOSTILBE \\ AND SERRAPINNUS SPECIES (CHARACIDAE: CHEIRODONTINAE) FROM \\ PARAGUAY RIVER BASIN, BRAZIL
}

\author{
Waldo Pinheiro Troy ${ }^{1}$, Érica Baleroni Pacheco ${ }^{2}$, Claudio Oliveira ${ }^{3}$, \\ Carlos Suetoshi Miyazawa ${ }^{4}$ \\ ${ }^{1}$ Universidade do Estado de Mato Grosso (UNEMAT), campus de Tangará da Serra, MT. \\ E-mail: waldotroy@gmail.com \\ ${ }^{2}$ Instituto Federal de Educação Ciência e Tecnologia (IFMT), campus de Campo Novo \\ dos Parecis, MT \\ ${ }^{3}$ Universidade Estadual Paulista Júlio de Mesquita Filho (UNESP), campus de Botucatu, \\ SP \\ ${ }^{4}$ Universidade Federal do ABC (UFABC), campus de Santo André, SP
}

Recebido para publicação em 12/05/2010

Aceito para publicação em 21/06/2010

\section{RESUMO}

Cheirodontinae constitui um grupo de 17 gêneros e 55 espécies ainda pouco estudadas citogeneticamente, sendo que a maioria dos dados disponíveis na literatura é da bacia do Alto Paraná. No presente estudo são caracterizadas cromossomicamente as espécies Odontostilbe pequira, Serrapinnus kriegi, Serrapinnus calliurus e Serrapinnus microdon da bacia do Alto Paraguai. O número diplóide modal $2 \mathrm{n}=52$ encontrado para estas espécies, bem como a maioria dos dados citados na literatura, evidencia um conservadorismo do número cromossômico para o grupo.

Palavras-chave: Citotaxonomia, macroestrutura cariotípica, Pantanal.

\begin{abstract}
Cheirodontinae constitutes a group of 17 genera and 55 species still poorly studied cytogenetically, being that most data available in the literature is for the Alto Paraná basin. In this study Odontostilbe pequira, Serrapinnus kriegi, Serrapinnus calliurus and Serrapinnus microdon from Alto Paraguay basin was characterized cytogenetically. The modal diploid number $2 n=52$ found for these species, as well as most of the data cited in the literature, is an evidence of a conservadorism in chromosome number for the group.
\end{abstract}

Keywords: Cytotaxonomy. Karyotypic macrostructure. Pantanal 


\section{Introdução}

A subfamília Cheirodontinae é composta por espécies de peixes de tamanho diminuto, sendo a maioria com até $5 \mathrm{~cm}$ de comprimento padrão. Estão distribuídas em 17 gêneros e 55 espécies que demonstram como principais características:

(1) apenas uma fileira de dentes pentacuspidados ou pedicelados no pré-maxilar; (2) ausência de musculatura na região cranial da bexiga natatória, caracterizando assim o pseudotímpano;

Tabela 1 - Revisão de dados encontrados sobre a macroestrutura cariotípica para a subfamília Cheirodontinae. $\mathrm{NF}=$ número fundamental. Referências bibliográficas: 29 . Wasko et al., 2001; 23. Paiva (2007); 26. Santi-Rampazzo et al., 2007.

\begin{tabular}{|c|c|c|c|c|c|}
\hline Espécie & $\begin{array}{l}\text { Número } \\
\text { diplóide } \\
\text { modal }\end{array}$ & Fórmula Cariotípica & $\mathrm{NF}$ & $\begin{array}{l}\text { Sistema de } \\
\text { Cromossomos } \\
\text { Sexuais }\end{array}$ & $\begin{array}{l}\text { Referência } \\
\text { Bibliográfica }\end{array}$ \\
\hline Odontostilbe paranensis & 52 & $\begin{array}{l}37 \mathrm{~m} / \mathrm{sm}, 12 \mathrm{st}, 3 \mathrm{a}(+) \\
36 \mathrm{~m} / \mathrm{sm}, 12 \mathrm{st}, 4 \mathrm{a}\left({ }^{7}\right)\end{array}$ & $\begin{array}{l}89 \\
88\end{array}$ & $\mathrm{ZZ/ZW}$ & 29 \\
\hline Serrapinnus notomelas & 52 & $10 \mathrm{~m}, 32 \mathrm{sm}, 6 \mathrm{st}, 2 \mathrm{a}$ & 100 & Não relatado & 23 \\
\hline Serrapinnus notomelas & 52 & $\begin{array}{c}10 \mathrm{~m}, 30 \mathrm{sm}, 8 \mathrm{st}, 2 \mathrm{a} \\
16 \mathrm{~m}, 23 \mathrm{sm}, 10 \mathrm{st}, 3 \mathrm{a}\end{array}$ & 100 & Não relatado & 23 \\
\hline Serrapinnus notomelas & 52 & $16 \mathrm{~m}, \underset{\left({ }^{3}\right)}{22 \mathrm{sm}, 10 \mathrm{st}, 4 \mathrm{a}}$ & 100 & $\mathrm{ZZ/ZW}$ & 26 \\
\hline Serrapinnus sp. 1 & 52 & $\begin{array}{l}8 \mathrm{~m}, 15 \mathrm{sm}, 4 \mathrm{st}, 25 \mathrm{a}(+) \\
8 \mathrm{~m}, 16 \mathrm{sm}, 4 \mathrm{st}, 24 \mathrm{a}\left({ }^{\Uparrow}\right)\end{array}$ & $\begin{array}{l}79 \\
80\end{array}$ & $\mathrm{ZZ/ZW}$ & 26 \\
\hline $\begin{array}{l}\text { Holoshestes heterodon } \\
\text { (=Serrapinnus heterodon) }\end{array}$ & 52 & 37m/sm, 12st, 3a (+) & 89 & $\mathrm{ZZ/ZW}$ & 29 \\
\hline
\end{tabular}

(3) ausência de mancha umeral e, (4) alto número de raios procurrentes da nadadeira caudal (MALABARBA, 1998, 2003; MALABARBA et al., 2004, BÜHRNHEIM; MALABARBA, 2006, 2007).

Estão distribuídos nas Américas Central e do Sul, ocorrendo desde a Costa Rica até o Chile central e Argentina, sendo que para a bacia do Alto Paraguai são descritos apenas dois gêneros, Serrapinnus e Odontostilbe, e cinco espécies: S. calliurus, S. kriegi, S. microdon, O. pequira, O. paraguayensis (MALABARBA, 2003).

No que diz respeito à citogenética, é uma subfamília ainda pouco estudada e os trabalhos existentes estão centrados na bacia do Alto Paraná, não sendo relatada variação do valor diplóide modal de $2 n=52$ cromossomos, mas sim modificações na macroestrutura cariotípica das espécies (OLIVEIRA et al. 1988; WASKO et al., 2001; PAIVA, 2007; SANTI-RAMPAZZO et al., 2007, entre outros). A Tabela 1 apresenta uma síntese dos dados disponíveis na literatura a respeito da macroestrutura cariotípica de diferentes espécies de Cheirodontinae.
Este trabalho tem por objetivo caracterizar cromossomicamente quatro espécies da subfamília Cheirodontinae coletados na bacia do Alto Paraguai. 


\section{Resultados}

Em Odontostilbe pequira foi encontrado o número diplóide modal $2 \mathrm{n}=52$ cromossomos, sendo a fórmula cariotípica formada por $24 \mathrm{~m}, 12 \mathrm{sm}, 12 \mathrm{st}$, 4a (Figura 1A) e número fundamental $(\mathrm{NF})=100$. O conjunto cromossômico de Serrapinnus kriegi foi constituído por 52 cromossomos, sendo $24 \mathrm{~m}, 18 \mathrm{sm}$, 10st (Figura 1B) e NF=104. S. calliurus apresentou $2 \mathrm{n}=52$ cromossomos, sendo $36 \mathrm{~m}, 12 \mathrm{sm}, 6 \mathrm{st}$ (Figura 1C) e NF=104. S. microdon apresentou um cariótipo com $2 \mathrm{n}=52$ cromossomos, sendo $30 \mathrm{~m}, 12 \mathrm{sm}, 8 \mathrm{st}$ e 4a (Figura 1D) e NF=100.
$\mathrm{O}$ número diplóide de $2 \mathrm{n}=52$ cromossomos encontrado no presente estudo em Serrapinnus calliurus, S. kriegi, S. microdon e Odontostilbe pequira corrobora os dados das espécies já estudadas da subfamília Cheirodontinae (WASKO et al., 2001; PAIVA, 2007; SANTI-RAMPAZZO et al., 2007), sendo sugerido por Arefjev (1990) como número basal para Characidae. A presença deste número cromossômico em todas as espécies já analisadas citogeneticamente reflete um conservadorismo para a subfamília, tal como ocorre em outros grupos da família Characidae, como Bryconinae (BIGONI et al. 1993; MARGARIDO \& GALETTI Jr., 1996;

Figura 1 - Cariótipos submetidos à coloração convencional por Giemsa de: (A) Odontostilbe pequira do rio Cuiabá, MT; (B) fêmea de Serrapinnus kriegi do rio Cuiabá, MT; (C) Serrapinnus calliurus do rio Bento Gomes, MT e; (D) macho de Serrapinnus microdon do rio Bento Gomes, MT (barra $5 \mu \mathrm{m})$.

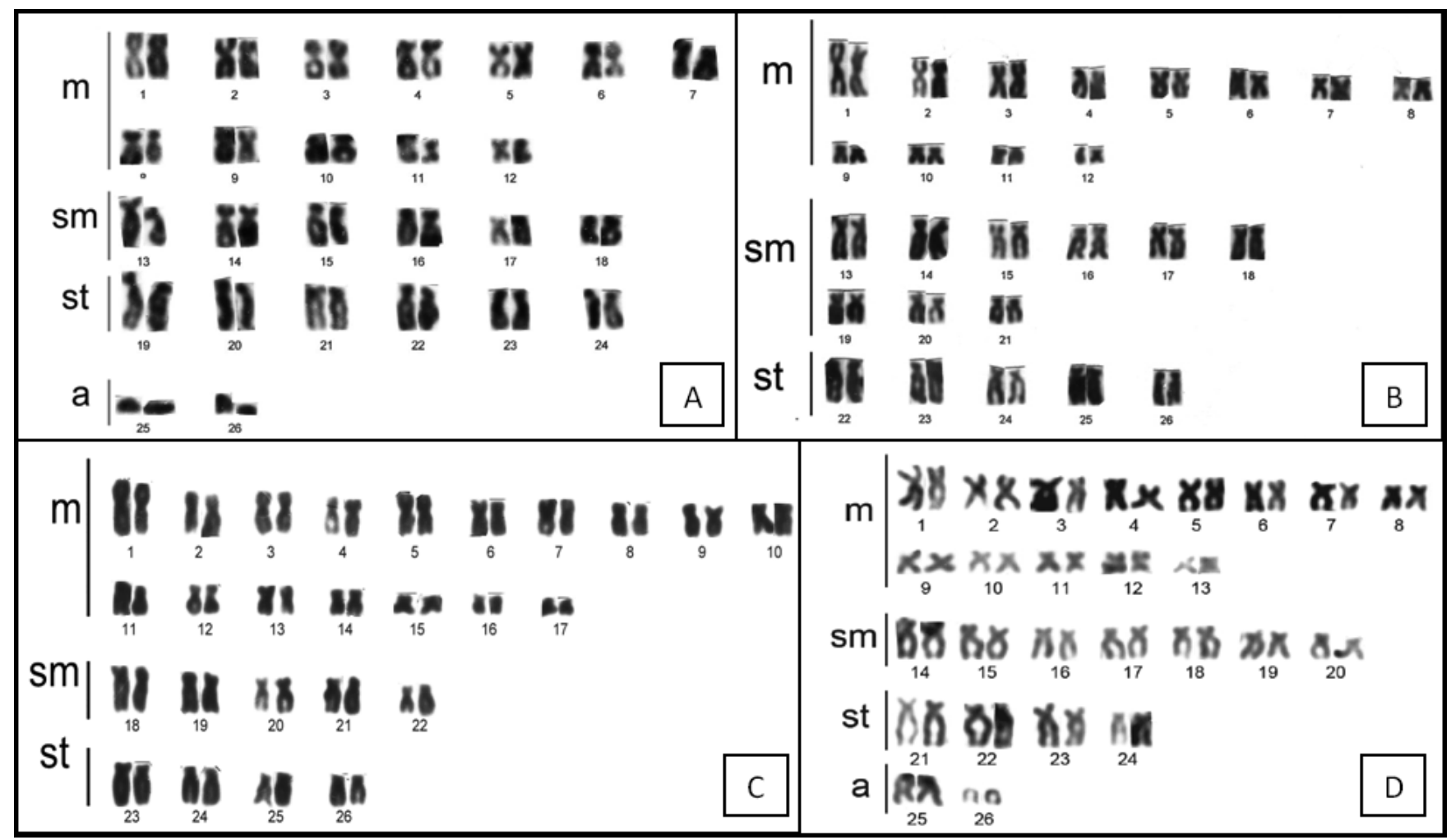

\section{Discussão}

Os caracídeos são largamente estudados no que diz respeito à citogenética, entretanto, os Cheirodontinae são uma exceção, provavelmente devido ao tamanho diminuto da maioria de suas espécies.
WASKO; GALETTI Jr., 2000; MARIGUELA et al., 2010), Stethaprioninae (PFISTER et al. 1997; FREITAS et al. 1998; CARVALHO et al., 2002), e em Oligosarcus (incertae sedis) (FALCÃO; BERTOLLO, 1985; OLIVEIRA et al., 1988; RUBERT; MARGARIDO, 2007), onde o número diplóide de $2 \mathrm{n}=50$ cromossomos é predominante. 
Por outro lado, na família Characidae têm sido observados freqüentemente exemplos de grupos com grande variação cariotípica, tal como Astyanax onde o número diplóide modal varia de $2 \mathrm{n}=36$ a $2 \mathrm{n}=50$ cromossomos (MORELLI et al., 1983; MOREIRA-FILHO; BERTOLLO, 1991; SOUZA; MOREIRA-FILHO, 1995; MAISTRO et al., 2000; CARVALHO et al., 2002) e Serrassalminae com variação de $2 n=54$ a 64 cromossomos (MURAMOTO et al., 1968; CESTARI; GALETTI Jr., 1992; NAKAYAMA et al., 2001, 2002 e NIRCHIO et al., 2002).

Apesar do conservadorismo apresentado em nível de número diplóide, os dados citogenéticos para a subfamília Cheirodontinae apontam para uma grande variação da macroestrutura cariotípica entre as espécies, o que é refletido nas fórmulas cariotípicas e no número fundamental (NF) que variou de $\mathrm{NF}=79 / 80$ em Serrapinnus sp. 1 (SANTI-RAMPAZZO et al., 2007) e NF=104 em Serrapinnus kriegi e Serrapinnus microdon (presente estudo), como pode ser visto na tabela 1. Possivelmente, durante a evolução destes cariótipos, estas variações da macroestrutura cromossômica estiveram relacionadas à rearranjos cromossômicos como as inversões pericêntricas e/ou paracêntricas, pois estas alteram a forma dos cromossomos sem alterar o número do conjunto cromossômico.

Na maioria das espécies estudadas até o momento da subfamília Cheirodontinae é freqüente a ocorrência do sistema de cromossomos sexuais do tipo ZZ/ZW, como em Holoshestes heterodon (=Serrapinnus heterodon) (WASKO et al., 2001), Serrapinnus sp.1 (SANTI-RAMPAZZO et al., 2007) e Serrapinnus notomelas (SANTI-RAMPAZZO et al., 2007) (tabela 1). A ausência deste sistema sexual nas espécies analisadas no presente estudo reflete a necessidade do aumento no número de indivíduos analisados de ambos os sexos, ainda que Paiva (2007), estudando Serrapinnus notomelas da bacia do Alto Paraná, não relate a presença de cromossomos sexuais. Por outro lado, Santi-Rampazzo et al. (2007) descreve a presença dos cromossomos sexuais do tipo ZZ/ZW para esta mesma espécie também para a bacia do Alto Paraná. Tal discordância entre os autores pode estar demonstrando uma divergência de identificação dos cromossomos, de identificação da espécie ou ainda a ocorrência de um complexo de espécies erroneamente identificado como uma única espécie.

\section{Conclusão}

A análise citogenética realizada até o momento em diferentes espécies da subfamília Cheirodontinae mostra certo conservadorismo do número diplóide modal, visto que as espécies analisadas até o momento apresentam número diplóide modal de $2 n=52$ cromossomos. Assim, estes dados demosntram que os grupos estudados devam pertencer a um grupo monofilético, sendo, entretanto, a distinção entre espécies e gêneros restrita à fórmula cariotípica, o que deverá ser investigado com a análise de mais espécies do grupo.

Agradecimentos: Os autores são gratos ao Fundo de Amparo à Pesquisa de Mato Grosso (FAPEMAT), pelo apoio financeiro.

\section{REFERÊNCIAS}

AREFJEV, V. A. Problems of karyotypic variability in the family Characidae (Pisces, Characiformes) with the description of the somatic karyotypes for six species of tetras. Caryologia, v. 43, p. 305-319, 1990.

BERTOLLO, L. A. C.; TAKAHASHI, C. S.; MOREIRA-FILHO, O. Considerações citotaxonômicas sobre Hoplias lacerdae (Pisces, Erythrinidae). Rev Genet Brasil. v. 1, p.103-120, 1978.

CARVALHO, M.L.; OLIVEIRA, C.\&FORESTI, F.Cytogenetic analysis of five species of the subfamily Tetragonopterinae (Teleostei, Characiformes, Characidae). Caryologia, Edizioni Polistampa, v. 55, n. 3, p. 181-188, 2002.

CESTARI, M. M.; GALETTI Jr, P. M. Chromosome evolution in the genus Serrasalmus and cytotaxonomic considerations about Serrasalminae (Characidae, Pisces). Brazil. J. Genet., v.15, p. 555-567, 1992.

FAlCÃO, J. N.; BERTOLlO, L. A. C. Chromosome characterization in Acestrorhynchinae andCynopotaminae (Pisces, Characidae). J. Fish Biol., v. 27, p. 603-610, 1985. 
FREITAS, P. D.; NAVARRETE, C.; MIYAZAWA, C. S.; GALETTI Jr, P. M. Chromosome characterization of a Neotropical Fish Poptella paraguayensis from Paraguay River Basin (Stethaprioninae, Characidae). Cytologia, The Japan Mendel Society, v. 63, p. 73-77, 1998.

LEVAN, A.; FREDGA, K.; SANDBERG, A. A. Nomenclature for centromeric position on chromosomes. Hereditas, n.52, p.201-220, 1964.

MAISTRO, E.D., OLIVEIRA, C.; FORESTI, F. Sympatric occurrence of two cytotypes of Astyanax scabripinnis (Characiformes, Characidae). Genetics and Molecular Biology, v.23, n.2, p.365-369, 2000.

MALABARBA, L. R. Monophyly of the Cheirodontinae, characters and major clades (Ostariophysi: Characidae). In: MALABARBA, L. R., REIS, R. E., VARI, R. P., LUCENA, Z. M., LUCENA, C. A. (Eds.). Phylogeny and classification of neotropical fishes. Porto Alegre, EDIPUCRS, 1998. p.193-233.

MALABARBA, L. R. Subfamily Cheirodontinae (Characins: tetras). In: KULLANDER, S.O.; FERRARIS, C.; REIS, R.E. (Eds). Check list of the freshwater fishes of South and Central America (CLOFFSCA). Porto Alegre: EDIPUCRS, 2003. p.215-221.

MARGARIDO, V. P.; GALETTI Jr, P. M. Chromosome studies in fish of the genus Brycon (Characiformes, Characidae, Bryconinae). Cytobios, v.85, p. 219-228, 1996.

MARIGUELA, Tatiane Casagrandre. Análise das relações filogenéticas entre os gêneros de Cheirodontinae (Ostariophysi: Characiformes: Characidae) utilizando seqüências de DNA mitocondrial e nuclear. Botucatu, 2009. 154p. Tese (Doutorado em) - Universidade Estadual Paulista.

MALABARBA, L. R.; LIMA, F. C. T.; WEITZMAN, S. H. A new species of Kolpotocheirodon (Teleostei: Characidae: Cheirodontinae: Compsurini) from Bahia, northeastern Brazil, with a new diagnosis of the genus. Proceedings of the Biological Society of Washington, v. 117, n. 3, p. 317-329, 2004.

MIYAZAWA, C. S.; GALETTI Jr, P. M. First cytogenetical studies in Characidium Species (pisces: characiformes, Characidiinae). Cytologia, The Japan Mendel Society, v. 59, p. 73-79, 1994.

MORELli, S.; BERTOLlO, L. A. C.; FORESTI, F.; MOREIRA-FILHO, O.; TOLEDO FILHO, S. A. Cytogenetic consideration on the genus Astyanax (Pisces, Characidae).I. Kariotypic variability. Caryologia, v. 36, n. 3, p.245-251, 1983.

MOREIRA-FILHO, O.; BERTOLLO, L. A. C. Astyanax scabripinnis (PISCES,CHARACIDAE): a species complex.

Rev. Bras. Genet, v.14, n.2, p.331-357, 1991.
MURAMOTO, J.; OHNO, S.; ATKIN, N. B. On the diploid state of fish order Ostariophysi. Cromossoma (Berl.), v. 24, p.59-66, 1968.

NAKAYAMA C.; JÉGU, M.; PORTO, J. I. R.; FELDBERG, E. Karyological evidence for a cryptic species of Piranha within Serrasalmus rhombeus (Characidae, Serrasalminae) in the Amazon. Copeia, v. 101, p.866-869, 2001.

NAKAYAMA C.; PORTO, J. I. R.; FELDBERG, E. A comparative cytogenetic study of five piranha species (Serrasalmus, Serrasalminae) from the Amazon basin. Genetica, v.114, p. 231-236, 2002.

NIRCHIO, M.; GRANADO, A., RON, E.; PÉREZ, J.E. Karyotype and Nucleolar Organizer Regions in Serrasalmus rhombeus (Linneaus, 1766) (Serrasalminae) from Caicara del Orinoco, Venezuela. Interciencia, v.27, p.676-678, 2002.

OLIVEIRA, C.; ALMEIDA-TOLEDO, L. F.; FORESTI, F.; BRITSKI, H. A.; TOLEDO-FILHO, S. A. Chromosome formulae of neotropical freshwater fishes. Rev. Bras. Genet, v. 11, n.3, p.577-624, 1988.

PAIVA, L. R. S. Citogenética de populações de Serrapinnus notomelas (Characidae: Cheirodontinae) da Bacia do Rio Tietê. 2007. 74p. Dissertação (Mestrado em Ciências Biológicas - Zoologia) - Universidade Estadual Paulista. Botucatu, 2007.

PFISTER, S. C.; MOREIRA-FILHO, O.; BERTOLLO, L. A. C. Cytogenetic studies in Orthospinus franciscencis (Pisces, Characidae, Stethaprioninae) and some considerations about karyotype evolution of the group. Cytobios, Inglaterra, v. 91, p. 97-101, 1997.

RUBERT, M.; MARGARIDO, V. P. Cytogentic studies in three species of the genus Oligosarcus. Brazilian Archives of Biology and Technology, v.50, n.1, p.127-135, 2007.

SANTI-RAMPAZZO, A. P.; NISHIYAMA, P. B; FERREIRA, P. E. B.; MARTINS -SANTOS, I. C. Cytogenetic analysis and description of the sexual chromosome determination system ZZ/ZW of species of the fish genus Serrapinnus (Characidae, Cheirodontinae). Genet. Mol. Res, v.6, n.3, p.504-509, 2007.

SOUZA, I. L.; MOREIRA-FILHO, O. Cytogenetic diversity in the Astyanax scabripinnis species complex (Pisces, Characidae) I. Allopatric distribution in a small stream. Cytologia, v.60, p.1-11, 1995.

WASKO, A. P.; GALETTI Jr,, P. M. Mapping 18S ribosomal genes in fish of the genus Brycon (Characidae) by fluorescence in situ hybridization (FISH). Genetics and Molecular Biology, v. 23, n. 1, p. 135-138, 2000.

WASKO, A. P.; CESAR, A. C. G.; MARTINS, C.; GALETTI Jr., P. M. AZZ/ZW sex chromosome system in Cheirodontinae fish. Chromosome Science, v.5, p.145-148, 2001. 